\title{
The effects of dual anti-platelet therapy on titanium implant osseointegration: an experimental study
}

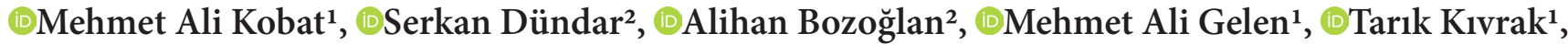 \\ ๑ Gökhan Artaş3, @Suna Aydın ${ }^{4}$
}

${ }^{1}$ Firat University, Faculty of Medicine, Department of Cardiology, Elazığ, Turkey

${ }^{2}$ Firat University, Faculty of Dentistry, Department of Periodontology Elazığ, Turkey

${ }^{3}$ Firat University, Faculty of Medicine, Department of Pathology, Elazı̆̆, Turkey

${ }^{4}$ Fethi Sekin City Hospital, Department of Cardiovascular Surgery, Elazığ, Turkey

Cite this article as: Kobat MA, Dündar S, Bozoğlan A, et al. The effects of dual anti-platelet therapy on titanium implant osseointegration: an experimental study. J Health Sci Med 2022; 5(1): 144-149.

\begin{abstract}
Aim: In this study, we aim to investigate the effect of dual anti-platelet agents on osseointegration by studying a sample of rats with titanium implants in their tibias.

Material and Method: The titanium implants were placed surgically to the left tibias of a sample group of 50 rats. After implantation, the rats were randomly divided into five groups: acetylsalicylic acid (ASA) ( $\mathrm{n}=10)$, treated with $20 \mathrm{mg} / \mathrm{kg}$ of ASA; ASA+ clopidogrel (CLPD) ( $\mathrm{n}=10$ ), treated with $20 \mathrm{mg} / \mathrm{kg}$ of ASA and $30 \mathrm{mg} / \mathrm{kg}$ of CLPD; ASA+ prasugrel (PRSG) $(\mathrm{n}=10)$, treated with $20 \mathrm{mg} / \mathrm{kg}$ of ASA and $15 \mathrm{mg} / \mathrm{kg}$ of PRSG; ASA+ ticagrelor (TCGR) $(\mathrm{n}=10)$, treated with $20 \mathrm{mg} / \mathrm{kg}$ of ASA and $300 \mathrm{mg} / \mathrm{kg}$ of TCGR; and a control group $(\mathrm{CNT})(\mathrm{n}=10)$ received no further treatment following implant surgery. The experimental period lasted four weeks, during which all medications were administered with oral gavage. Concluding the experimental period, the animals were euthanized, and researchers collected blood serums and the implants, along with some surrounding bones, from each rats.

Results: Bone implant connection and bone filling ratios (\%) were observed histologically and documented. The bone-implant connection and bone filling ratios of the rats do not show statistically significant differences between the groups examined $(\mathrm{P}>0.05)$.

Conclusion: In this study, it was shown that there was no bone healing problem between the antiaggregant given groups and between these groups and the control group. Too many people have to take single or double antiaggregant due to many diseases. We think that it is an important study in terms of knowing that bone healing will not be negatively affected when dental implants or other bone prosthesis procedures are applied to these patients. The results of this study should be supported by further research.
\end{abstract}

Keywords: Anti-platelet drugs, bone healing, bone-implant connection, dental implant osseointegration, dual antiplatelet theraphy

\section{INTRODUCTION}

Dual anti-platelet therapy (DAPT) consists of a combination of acetylsalicylic acid (ASA) and an oral P2Y12 inhibitor (clopidogrel, ticagrelor or prasugrel), and forms the basis of antithrombotic therapy after myocardial infarction (MI) or percutaneous coronary intervention (PCI)(1). In Europe, many older adults are DAPT patients, and their number is growing steadily. According to data from 2015, DAPT was in use for 1,400,000 PCI patients and 2,200,000 MI patients annually throughout Europe (2). DAPT helps prevent atherothrombotic events by reducing platelet aggregation. ASA, the primogenital anti-platelet agent, has been shown to reduce the incidence of recurrent major adverse cardiovascular events by $20 \%$ (3).

Acetylsalicylic acid irreversibly inhibits thromboxane A2 production by inhibiting cyclooxygenase 1 . The available evidence suggests that $75-100 \mathrm{mg} /$ day of ASA be prescribed daily for every patient with coronary artery disease (CAD), whether or not there is a history of MI (3). It is also recommended for use in the prevention of clot formation in other situations (4).

Clopidogrel is a thienopyridine derivative that is the same as prasugrel, which irreversibly blocks the P2Y12 
adenosine diphosphate (ADP) receptor on platelets. This reduces platelet activation and aggregation, ultimately decreasing blood viscosity and increasing bleeding time (5). Clopidogrel is recommended in addition to ASA after coronary stenting, regardless of the type of stent, in a dosage of $75 \mathrm{mg}$ daily for six months after proper loading (e.g. $600 \mathrm{mg}$ loading or $75 \mathrm{mg}$ therapy for five days). In cases of life-threatening bleeding or high bleeding risk, one to three months' treatment may be recommended (6).

Prasugrel like clopidogrel, prasugrel is an irreversible P2Y12 antagonist of the thienopyridine class; however, in vitro studies have shown it to be both faster and stronger (7). In patients with acute coronary syndrome (ACS) who undergoing PCI, prasugrel (60 mg loading dose, 10 $\mathrm{mg}$ /day maintenance dose) is primarily recommended in addition to ASA, according to ESC guidelines, unless there are contraindications (8).

Ticagrelor is a thienopyridine-group drug that blocks the P2Y12 receptor on the platelet, reversibly inhibiting platelet aggregation. Since it does not require metabolic activation, it is more effective than clopidogrel $(9,10)$. In patients with ACS, ticagrelor (180 mg loading dose, $90 \mathrm{mg}$ twice daily) with ASA is recommended, even if treatment with clopidogrel has, unless there are contraindications (11). Although ASA and clopidogrel are sometimes used alone, a combination of clopidogrel, ticagrelor or prasugrel with ASA is recommended after coronary artery disease, ACS and coronary stenting (6).

Dental implant-supported prostheses are a scientifically proven treatment option for people with dental deficiencies like edentulism. Many factors are known to affect wound and bone healing. As for osseointegration, defined as the integration of bones and implants, the main factors impacting its success are the quality and amount of bone placed in the dental implant; the patient's systemic diseases, smoking and drug use or lack thereof; and the geometrical and surface features of the implant device $(12,13)$. Osseointegration is critical for a dental implant's success. Research and technological developments in the field of oral implantology have focused on the points affecting osseointegration. Bone healing is principal in dental implant surgery, as in all branches of bone surgery. Osseointegration and the quality of peri-implant bone tissue play a guiding role in the dental implant-supported prosthetic treatment. Infections, loss of function, and pain are a few of the problems that could lead to the failure of the implant during the bone healing process. On the other hand, some circumstances and applications that can positively affect this process may contribute to more successful dental implant outcomes $(12,13)$.
To our knowledge, there are no previously existing studies evaluating the effects of dual anti-platelet therapy on osseointegration. However, controversial studies have continues to suggest the impact of antiaggregant use on bone healing. As such, identifying pharmacological agents that can affect bone healing continues to be a current issue. This study aims to evaluate the effects of systemic anti-platelet applicaitons on the osseointegration of titanium implants placed in rat tibias.

\section{MATERIAL AND METHOD}

\section{Animals and Experimental Design}

All experimental and surgical procedures in this study were performed in Elazığ, Firat University Experimental Research Center in Elazığ, Turkey. The study was approved by the Firat University Animal Experimental Ethics Committee on January 10, 2020 (Protocol Number: 371066). All procedures were performed adhered to the ethical rules and principles of the Helsinki Declaration.

In this study, 50 healthy adult female Sprague-Dawley rats aged 3-3.5 months were used. The average weight of each rat at the start of the experiment was 240-260 grams. The rats were kept in plastic containers, whose temperatures were checked daily. During the experiment, the rats were not limited in terms of food and water, and the light cycle in the lab was adjusted to rotate through 12 hours of darkness and 12 hours of brightness. All of the rats were selected in the same estrus period to ensure standardization throughout the experimental protocols (13).

Titanium implants (Implance Dental Implant System, AGS Medical Corporation, İstanbul, Turkey,) were surgically inserted into the rats' tibias, following which they were randomly divided into five groups with similar mean weights in each group: the control (CNT) group $(n=10)$, the ASA group $(n=10)$, the ASA+Clopidogrel (ASA+CLPD) group $(n=10)$, the $\mathrm{ASA}+$ Prasugrel (ASA+PRSG) group $(\mathrm{n}=10)$ group, and the ASA+Ticagrelor (ASA+TCGR) $(n=10)$ group. In the CNT group, the rats received no further treatment during the experimental period following implant surgery. In the experimental ASA group, they were given $20 \mathrm{mg} / \mathrm{kg}$ per day of ASA; in the ASA+CLPD group, they were given $20 \mathrm{mg} / \mathrm{kg}$ of ASA and $30 \mathrm{mg} / \mathrm{kg}$ of clopidogrel per day; in the ASA+PRSG group, they were given $20 \mathrm{mg} / \mathrm{kg}$ of ASA and $15 \mathrm{mg} / \mathrm{kg}$ of prasugrel per day; and in the ASA+TCGR group, they were given $20 \mathrm{mg} / \mathrm{kg}$ of ASA and $300 \mathrm{mg} / \mathrm{kg}$ ticagrelor per day. All medication was administered via oral gavage (14). Four weeks after implant placement, the animals were euthanized $(12,13)$. 


\section{Surgical Procedures}

All of the rats included in this study were given antibiotic prophylaxis intramuscular injections of 50 $\mathrm{mg} / \mathrm{kg}$ penicillin 30 minutes before general anesthesia. Xylazine (5 mg/kg, Rompun, Bayer, Germany) and Ketamine hydrochloride $(50 \mathrm{mg} / \mathrm{kg}$, Ketasol, Richter Pharma, Wels, Austria) were injected intramuscularly into the rats, and general anesthesia was accomplished. Much attention was paid to sterilization during all surgical procedures. After were anesthetized, the surgical site was shaved and cleaned with povidoneiodine. An incision approximately $10-15 \mathrm{~mm}$ in length was made along the left tibial crest. The soft tissues were separated, and the tibia metaphyseal bone was exposed. Implant beds were adjusted with a drill with saline perfusion. The titanium implant was then placed in the metaphyseal part of the left tibia with primarily stabilized. The titanium implants we used were 4 $\mathrm{mm}$ long and $2.5 \mathrm{~mm}$ in diameter (grade 4 titanium, machined surface, TiAI6V4). After the titanium implants were placed, the flaps were returned to their original positions, and the layers were sutured using vicryl 5-0 (12,13).

\section{Histologic Procedures and Analysis}

The original integrated implants and surrounding bone tissue were used for the subsequent histomorphometric and immunohistochemical analyses. All samples were kept in $10 \%$ formaldehyde when initially taken and then transferred to $10 \%$ formic acid after to soften the tissues. After the surrounding tissues were softened, the implants were carefully removed from the samples. The samples were dried and embedded in paraffin wax. Finally, they were staining with hematoxylin and eosin for microscopic analysis. Sections of $6 \mu \mathrm{m}$ in thickness, corresponding to the implants with surrounding bone tissue area, were evaluated by light microscopy (15).

Histological staining for bone-implant connection (BIC) and bone filling (BF) analysis was performed using the hematoxylin and eosin extracted from the samples. $\mathrm{BIC}$ and BF were examined using a light microscope and image analyzer at the Department of Pathology Laboratories, Faculty of Medicine, Firat University, Elazig, Turkey. Histomorphometry was performed by the same specialist using stereological software. The BIC ratio (or percentage) of each specimen was measured as the ratio of implant surface directly touching the bone compared to the total implant surface length. The BF ratio (or percentage) was measured by calculating the bone-filled area's distance $0.5 \mathrm{~mm}$ from the implant (the medial, distal and apical portion of the implant) $(12,13)$. Researchers used the Olympus DP71 software imaging system for the histological analysis.

\section{Biochemical Analysis}

While the rats were anesthetized, blood samples were obtained through cardiac puncture to measure the serum alkaline phosphatase (ALP), calcium $(\mathrm{Ca})$, phosphorus $(\mathrm{P})$, blood urea nitrogen $(\mathrm{BUN})$, creatinine (Cr), aspartate aminotransferase (AST), alanine aminotransferase (ALT), and magnesium $(\mathrm{Mg})$. Biochemical data were measured for each rat at the Central Biochemistry Laboratory of the Faculty of Medicine, Frrat University.

\section{Statistical Analysis}

Statistical analysis was performed using IBM SPSS Statistics (Version 22.0). The appropriateness of the normal distribution of the parameters was assessed by the Kolmogorov-Smirnov test. A one-way analysis of variance (one-way ANOVA) was performed for the parameters, showing a normal distribution. Tukey's Honestly Significant Difference test was used to detect which group caused the difference. Continuous data were presented as mean \pm standard deviation values, and numerical measurements were presented as median, minimum, and maximum values. A statistical significance level of $95 \%(\mathrm{P}<0.05)$ was adopted.

\section{RESULTS}

After the four-week experimental period, a rat in the ticagrelor group died. Apart from this, no systemic or wound-related problems were observed in the rats.

The BIC ratios of the rats in the ASA group were lower than those of the rats in the control and other groups, but not to a statistically significant extent $(\mathrm{P}>0.05)$ (Table 1). Statistically, significant differences were not detected between the groups in terms of their relative $\mathrm{BF}$ ratios $(\mathrm{P}>0.05)$ (Table 2). Biochemical results (BUN, Cr,C AST, ALT, Ca, ALP, $\mathrm{P}$ and $\mathrm{Mg}$ ) were similar between groups $(\mathrm{P}>0.05)$ (Table 3) (Figure 1).

\begin{tabular}{|lcc|}
\hline \multicolumn{3}{|l|}{ Table 1. Bone implant connection ratio (\%) of the groups } \\
\hline & Mean \pm Std. deviation & p \\
\hline Control $(\mathrm{n}=10)$ & $61.00 \pm 15.23$ & $>0.05$ \\
ASA $(\mathrm{n}=10)$ & $50.20 \pm 17.39$ & $>0.05$ \\
ASA+Klopidogrel $(\mathrm{n}=10)$ & $66.50 \pm 18.41$ & $>0.05$ \\
ASA+Prasugrel $(\mathrm{n}=10)$ & $63.00 \pm 14.94$ & $>0.05$ \\
ASA+ticagrelor $(\mathrm{n}=9)$ & $60.00 \pm 18.20$ & $>0.05$ \\
\hline
\end{tabular}

\begin{tabular}{|lcc|}
\hline \multicolumn{3}{|l|}{ Table 2. BF ratio (\%) of the groups } \\
\hline & Mean \pm Std. deviation & $\mathbf{p}$ \\
\hline Control $(\mathrm{n}=10)$ & $62.00 \pm 9.77$ & $>0.05$ \\
ASA $(\mathrm{n}=10)$ & $61.00 \pm 15.23$ & $>0.05$ \\
ASA+Klopidogrel $(\mathrm{n}=10)$ & $62.50 \pm 16.54$ & $>0.05$ \\
ASA+Prasugrel $(\mathrm{n}=10)$ & $60.50 \pm 15.17$ & $>0.05$ \\
ASA+ticagrelor $(\mathrm{n}=9)$ & $57.77 \pm 16.97$ & $>0.05$ \\
\hline
\end{tabular}




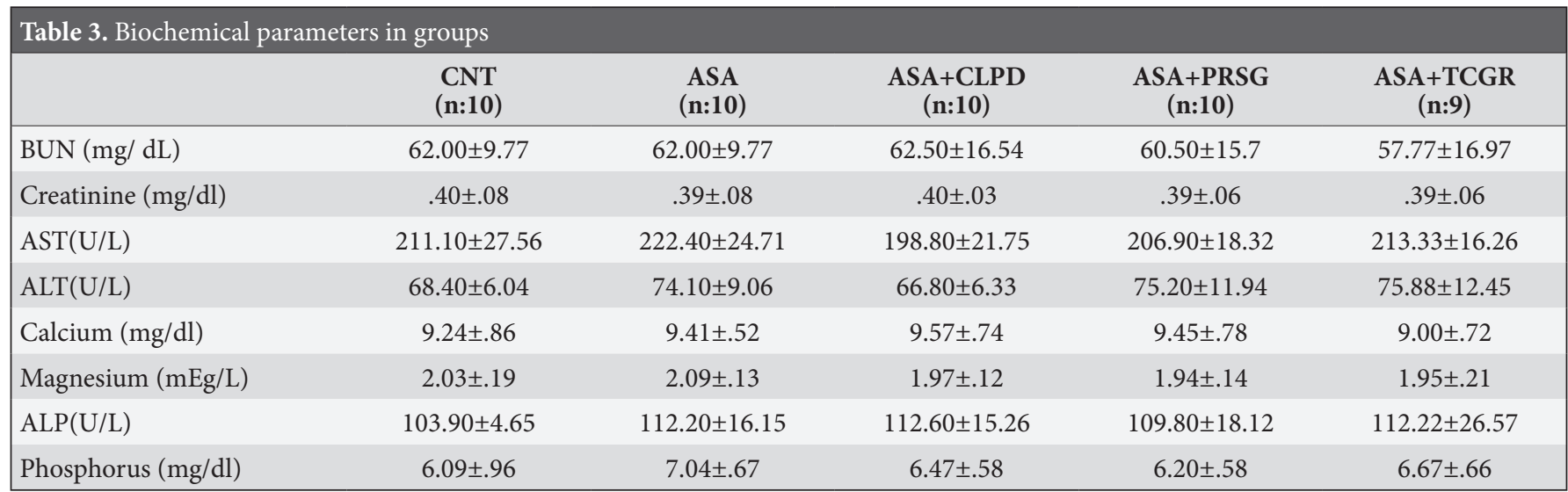
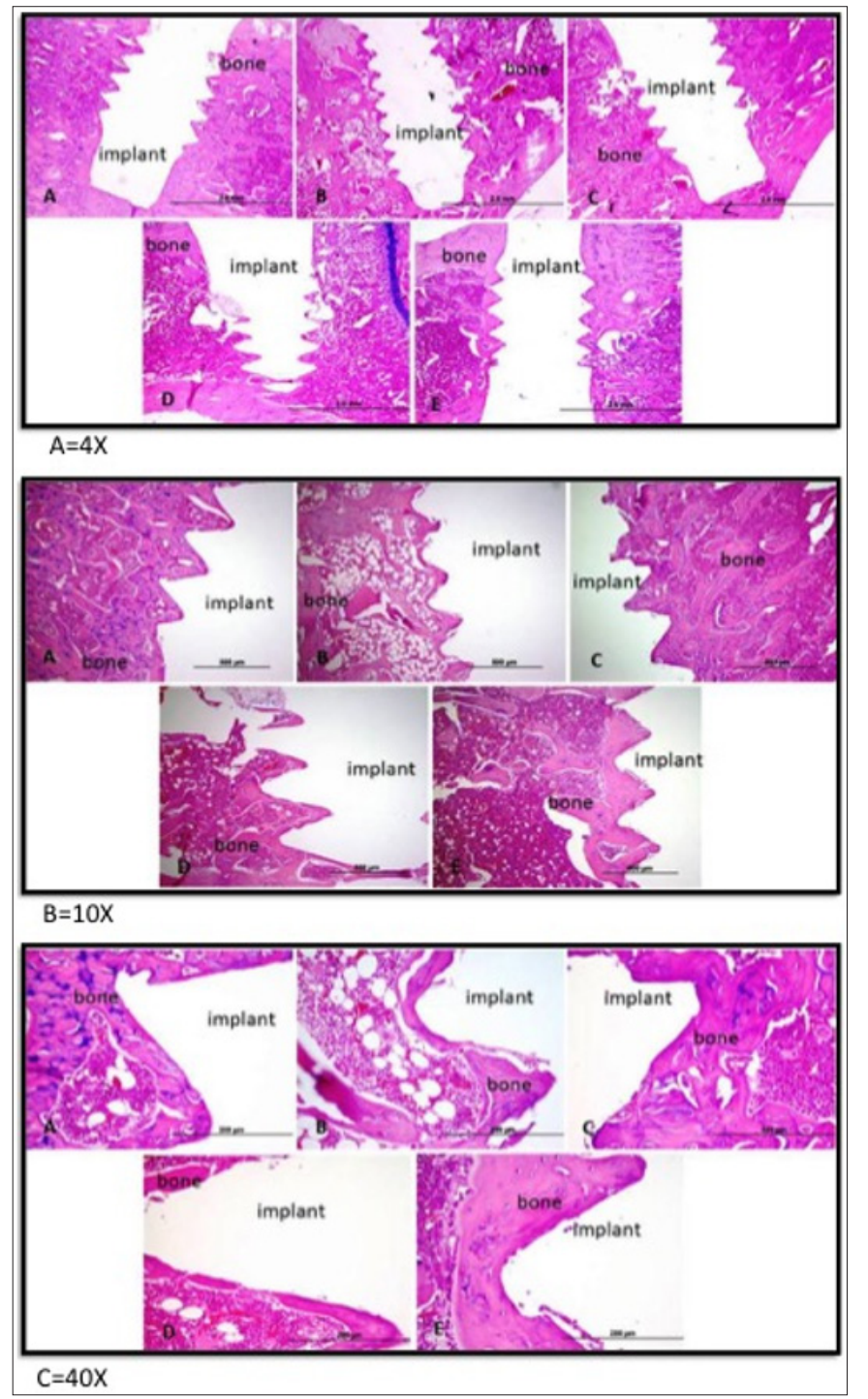

Figure 1. Decalcified histologic images of the A: Control B: Asetilsalisilic acid (ASA), C: ASA+Klopidegrol, D: ASA+Prasugrel, E: ASA+Ticagrelor Group, $(\mathrm{F}=4 \mathrm{X}$ magnification, Hematoxilen Eosine, $\mathrm{G}=10 \mathrm{X}$ magnification, Hematoxilen Eosine, $\mathrm{H}=40 \mathrm{X}$ magnification, Hematoxilen Eosine,). Implant surface not contacting bone $(\alpha)$, Implant surface in contacting with the bone $(\beta)$, Total implant surface: $\mathfrak{E}$, Bone Implant Contact Ratio (\%): $\mathfrak{E}-\alpha(\beta) / \mathfrak{E}$. Bone filling detected by measuring the bone filled areas distances $0.5 \mathrm{~mm}$ from implant (the medial, distal and apical portion of implants). Bone filling areas

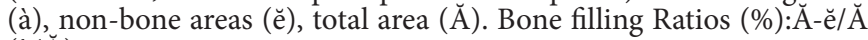
$(\grave{a} / \check{A})$.

\section{DISCUSSION}

Long-term ASA therapy is beneficial in reducing the risk of subsequent MI, stroke, and vascular death among patients with a wide range of cardiovascular disease symptoms; yet concerningly, Vestergaard P et al. (16) showed that patients using ASA experienced more bone fractures. This increased bone fracture rate was lower in people using low doses of ASA (17). In our study, while $\mathrm{BF}$ rates were similar, BIC rates were lower in the group receiving ASA compared to other groups, but the difference was not significant. The use of antiaggregant ASA $(75-325 \mathrm{mg} / \mathrm{d})$ was not found to have a negative effect on bone healing. Similarly, Hunter et al. (18) found no difference observed between bone healing of ankle fractures in the 6-month follow-up of patients using ASA postoperatively to treat their pain.

Different results have been obtained in previous studies on bone healing for patients taking clopidogrel. In our study, the osseointegration level in the ASA and clopidogrel group was similar to that of the control and other groups. Syberg et al. (19) showed that clopidogrel treatment in adult ovariectomized mice for four weeks resulted in a significant reduction of trabecular bone volume, alongside a reduction in trabecular number in tibial and femur bone tissues compared with control group animals. In contrast, Su et al. (20) reported that treating adult ovariectomized mice with high dosages of clopidogrel resulted in a significant increase in trabecular bone volume in the tibia and significantly decreased serum levels of osteoclast activity marker when compared to vehicle-treated control mice. Additionally, Lillis et al. (21) reported that bone healing was further along in a sample group of rabbits receiving six weeks of clopidogrel treatment for in circular calvarial defect.

In the group given ASA and prasugrel, the BIC and $\mathrm{BF}$ ratios were similar to the control group and other groups. There was no difference in terms of bone healing. There are no studies we could find in the literature investigating the effect of prasugrel or ASA+prasugrel on bone healing. 
In our study, no significant difference was observed between the group receiving ASA and ticagrelor and the control and other groups in terms of osseointegration. There is only one study we could find in the literature investigating the effect of ticagrelor on bone healing. Mediero et al. (22) showed that ticagrelor inhibited osteoclast differentiation and promoted osteoblast differentiation in vitro. Ticagrelor promotes bone formation by regulating osteoclast and osteoblast functions in vitro with an adenosine-induced mechanism.

CAD has reached almost epidemic rates in most societies and causes more deaths than all other cardiovascular disease groups (23). CAD and peripheral artery disease require the use of ASA for life. In addition, ASA is used in ischemic stroke, transient ischemic attack and preeclampsia treatments (4). The P2Y12 inhibitor(s) used with ASA after acute MI or PCI must be taken for a period of six months to one year. Meanwhile, dental implantation is a suitable treatment method for those who have lost teeth or who are completely toothless, and many patients who have planned to receive implant treatment use single or double antiaggregants.

Not knowing how bone healing will proceed due to possible negative interactions with other medication can cause hesitation and sometimes delay treatment for patients seeking implants. However, prior to our writing, there were no studies readily available which showed the presence or absence of a relationship between antiaggregant use and bone healing after the dental implant procedure.

We found no difference between the various antiaggregant agents as they related to the osseointegration of titanium implants in rat tibias, nor was there a significant difference when they were applied versus in the control group. This result will contribute to the literature and to clinical practice by allowing consulting surgeons to more comfortably proceed with dental implantation when patients are undergoing DAPT.

Our study has several limitations. First, anti-platelet was not applied before the procedure. Second, our use of rats is a necessarily imperfect analog for similar drug pathways and health outcomes in humans. Third, in this study, we could not investigate the effects of long-term anti-platelet use on bone healing since there was no longer follow-up. Finally, long bones (tibia-femur) and craniofacial bones (mandibula-maxilla) have different osteogenic potential; therefore, antiaggregant use may respond differently on craniofacial bones (13).

\section{CONCLUSION}

This study showed that daily anti-platelet uses during the 28-day experimental period did not affect bone-implant contact and bone filling ratios in for titanium implants in rat tibias. However, more extended studies are needed to clarify whether anti-platelet use affects osseointegration in the bones of the face and during longer periods of time.

\section{ETHICAL DECLARATIONS}

Ethics Committee Approval: The study was approved by the Firat University Animal Experimental Ethics Committee on January 10, 2020 (Protocol Number: 371066).

Informed Consent: Since the study was an animal experiment, approval was obtained from the animal experiment ethics committee.

Referee Evaluation Process: Externally peer-reviewed.

Conflict of Interest Statement: The authors have no conflicts of interest to declare.

Financial Disclosure: The authors declared that this study has received no financial support.

Author Contributions: All of the authors declare that they have all participated in the design, execution, and analysis of the paper and that they have approved the final version.

Akcnowledgement: Authors wish to thanks Implance Dental Implant System, AGS Medical Corporation, İstanbul, Turkey.

\section{REFERENCES}

1. Knuuti J, Wijns W, Saraste A, et al. 2019 ESC Guidelines for the diagnosis and management of chronic coronary syndromes. Eur Heart J 2020; ; 41: 407-77.

2. Population Division. Department of Economic and Social Affairs. United Nations. Revision of World Population Prospects https: // esa.un.org/unpd/wpp/.

3. Baigent C, Blackwell L, Collins R, et al. Aspirin in the primary and secondary prevention of vascular disease: collaborative metaanalysis of individual participant data from randomised trials. Lancet 2009; 373: 1849-60.

4. Henderson JT, Whitlock EP, O’Connor E, Senger CA, Thompson JH, Rowland MG. Low-dose aspirin for prevention of morbidity and mortality from preeclampsia: a systematic evidence review for the U.S. Preventive Services Task Force. Ann Intern Med 2014; 160: 695-703.

5. Kumar A, Cannon CP. Acute coronary syndromes: diagnosis and management, part I. Mayo Clin Proc 2009; 84: 917-38.

6. Valgimigli M, Bueno H, Byrne RA, et al. 2017 ESC focused update on dual anti- platelet therapy in coronary artery disease developed in collaboration with EACTS: The Task Force for dual anti-platelet therapy in coronary artery disease of the European Society of Cardiology (ESC) and of the European Association for CardioThoracic Surgery (EACTS). Eur Heart J 2018; 39: 213-60.

7. Brandt JT, Payne CD, Wiviott SD, et al. A comparison of prasugrel and clopidogrel loading doses on platelet function: magnitude of platelet inhibition is related to active metabolite formation. Am Heart J 2007; 153: 66.e9-e16.

8. Wiviott SD, Braunwald E, McCabe $\mathrm{CH}$, et al. Prasugrel versus clopi- dogrel in patients with acute coronary syndromes. N Engl J Med 2007; 357: 2001-15.

9. Storey RF. Pharmacology and clinical trials of reversibly-binding P2Y12 inhibitors. Thromb Haemost 2007; 105: S75-81. 
10.Dehghani P, Lavoie A, Lavi S, et al. Effects of ticagrelor versus clopidogrel on platelet func- tion in fibrinolytic-treated STEMI patients undergoing early PCI. Am Heart J 2017; 192: 105-12.

11. Wallentin L, Becker RC, Budaj A, et al. Ticagrelor versus clopidogrel in patients with acute coronary syndromes. N Engl J Med 2009; 361: 1045-57.

12. Bozoglan A, Dundar S, Yildirim TT, et al. Effects of different levels of restraint stress on bone-implant contact. J Craniofac Surg 2019; 30: 1294-7.

13.Dundar S, Bozoglan A, Bulmus O, et al. Effects of restraint stress and high-fat diet on osseointegration of titanium implants: an experimental study. Braz Oral Res 2020; 34: e008.

14. Birnbaum Y, Tran D, Chen H, Nylander S, Sampaio LC, Ye Y. Ticagrelor improves remodeling, reduces apoptosis, inflammation and fibrosis and increases the number of progenitor stem cells after myocardial infarction in a rat model of ischemia reperfusion. Cell Physiol Biochem 2019; 53: 961-81.

15. Artas G, Gul M, Acikan I, et al. A comparison of different bone graft materials in peri-implant guided bone regeneration. Braz Oral Res 2018; 32: 59.

16. Collaborative overview of randomized trials of anti- platelet treatment, I: prevention of vascular death, MI and stroke by prolonged anti-platelet therapy in different categories of patients. Anti-platelet Trialists Collaboration. Br Med J 1994; 308: 235-46.

17. Vestergaard P, Steinberg TH, Schwarz P, Jørgensen NR. Use of the oral platelet inhibitors dipyridamole and acetylsalicylic acid is associated with increased risk of fracture. Int J Cardiol 2012; 160: $36-40$

18. Hunter AM , Tyler PM , Charles CP, et al. Postoperative aspirin use and its effect on bone healing in the treatment of Ankle Fractures. Injury 2020; 51: 554-8.

19. Syberg S, Brandao-Burch A, Patel JJ, et al. Clopidogrel (Plavix), a P2Y12 receptor antagonist, inhibits bone cell function in vitro and decreases trabecular bone in vivo. J Bone Miner Res 2012; 27: $2373-86$

20.Su X, Floyd DH, Hughes A, et al. The ADP receptor P2RY12 regulates osteoclast function and pathologic bone remodeling. J Clin Invest 2012; 122: 3579-92.

21.Lillis T, Veis A, Sakellaridis N, Tsirlis A, Dailiana Z. Effect of clopidogrel in bone healing-experimental study in rabbits. World J Orthop 2019; 10: 434-45.

22. Mediero A, Wilder T, Reddy VS, et al. Ticagrelor regulates osteoblast and osteoclast function and promotes bone formation in vivo via an adenosine-dependent mechanism. FASEB J 2016; 30: 3887-900.

23. Wong ND. Epidemiological studies of CHD and the evolution of preventive cardiology. Nat Rev Cardiol 2014; 11: 276-89. 